\title{
Role of strain in polarization switching in semipolar InGaN/GaN quantum wells
}

\author{
Qimin Yan, ${ }^{1}$ Patrick Rinke, ${ }^{1,2}$ Matthias Scheffler, ${ }^{1,2}$ Chris G. Van de Walle ${ }^{1}$ \\ ${ }^{1}$ Materials Department, University of California, Santa Barbara, California 93106-5050, USA \\ ${ }^{2}$ Fritz-Haber-Institut der Max-Planck-Gesellschaft, Faradayweg 4-6, D-14195 Berlin, Germany
}

(Dated: March 9, 2011)

\begin{abstract}
The effect of strain on the valence-band structure of (11 $\overline{2} 2)$ semipolar InGaN grown on GaN substrates is studied. A $\mathbf{k} \cdot \mathbf{p}$ analysis reveals that anisotropic strain in the $c$-plane and shear strain are crucial for deciding the ordering of the two topmost valence bands. The shear-strain deformation potential $D_{6}$ is calculated for GaN and InN using density functional theory with the Heyd-ScuseriaErnzerhof hybrid functional [J. Heyd, et al., J. Chem. Phys. 124, 219906 (2006)]. Using our deformation potentials and assuming pseudomorphically strained structure, no polarization switching is observed. We investigate the role of partial strain relaxation in the observed polarization switching.
\end{abstract}

PACS numbers: $71.20 . \mathrm{Nr}, 71.70 . \mathrm{Fk}, 85.60 . \mathrm{Bt}$

Indium Gallium Nitride (InGaN) alloys are already widely used as commercial light emitters. ${ }^{1,2}$ For high efficiency devices semipolar or nonpolar quantum-well orientations have been proposed. In these orientations the effect of spontaneous and piezoelectric polarization is reduced and thereby the carrier overlap increased. ${ }^{3-5}$ Growth of InGaN on GaN along semipolar or nonpolar directions leads to different strain conditions than the conventional growth along the $c$-direction. This affects the splitting of the uppermost valence bands and hence the polarization of the emitted light. A corresponding optical anisotropy has already been observed for both nonpolar ${ }^{6-8}$ and semipolar ${ }^{9-14}$ devices. This raises the question of how to control the polarization of the emitted light, which is not only of scientific interest but also of technical importance for the performance of light-emitting diodes and laser diodes.

Recently, polarization switching has been reported for semipolar InGaN grown on a (1122) GaN substrate. ${ }^{11,12}$ The dominant polarization direction of emission was found to switch from [1100] (perpendicular to the $c$-axis) to [1123] when the In concentration was increased above $30 \%$, but the mechanism responsible for the change in polarization could not be identified. Other experimental studies have found no evidence of polarization switching, however. Under high excitation power density the sign of the polarization ratio of InGaN/GaN was found to remain unchanged even at high In content. ${ }^{13,14}$ Why the polarization ratio is affected by the excitation power density also remains unclear.

Three factors critically influence the band structure of InGaN quantum wells $(\mathrm{QW})$ and therefore the polarization of the emitted light: indium concentration, strain, and quantum confinement. Based on $\mathbf{k} \cdot \mathbf{p}$ modeling Yamaguchi predicted that the QW thickness would strongly affect the polarization. ${ }^{15}$ This seems qualitatively consistent with the results of Masui et al., who observed an enhancement in optical polarization for thinner quantum wells. ${ }^{12}$ However, in Yamaguchi's work the magnitude of this quantum confinement effect is very sensitive to the choice of Luttinger parameters ${ }^{16}$ and can range from 2 to $20 \mathrm{meV}$ for $2 \mathrm{~nm}$ thick quantum wells. Ueda et al., on the other hand, found no appreciable QW thickness effect. ${ }^{11}$ They proposed strain to be the dominant factor, and derived a large shear-strain deformation potential of $D_{6}=-8.8 \mathrm{eV}$ from their measurements. ${ }^{11}$

Strain in $(11 \overline{2} 2)$ semipolar InGaN grown on GaN substrates is characterized by shear strain and anisotropic strain in the $c$ plane. In this work, we calculate the effect of shear strain on the band structure of GaN and InN from first principles. We have previously reported a consistent set of strain deformation potentials $D_{1}$ to $D_{5}$ for the group-III nitrides, ${ }^{17}$ which is completed by the $D_{6}$ values reported here. With these deformation potentials and the band parameters of Ref.16 we use the $\mathbf{k} \cdot \mathbf{p}$ approach to determine the energies of the three highest valence bands at the $\Gamma$ point. For ideal (unrelaxed) strain conditions, we find no polarization switching for semipolar InGaN alloys grown on (112̄2) GaN substrates. Polarization switching can occur, however, when we assume the strain to be partially relaxed.

Our first-principles calculations are performed using the plane-wave projector augmented wave method (PAW) as implemented in the VASP code. ${ }^{18}$ We use the Heyd-Scuseria-Ernzerhof hybrid exchange-correlation functional ${ }^{19,20}$ with a screening parameter $\mu=0.2$ and a mixing parameter of $\alpha=0.25$ (HSE06). In previous work we showed that HSE06 gives accurate lattice parameters and band gaps in good agreement with experiment for the group-III nitrides. ${ }^{17}$ The semi-core $d$ electrons of Ga and In are treated as valence electrons. A $6 \times 6 \times 4 \Gamma$ point centered $k$-point sampling and a plane-wave cutoff of $600 \mathrm{eV}$ are used to ensure an accurate relaxation of the internal displacement parameter $u$.

The energies of the three highest valence bands at the $\Gamma$ point $\left(E_{1}\right.$ to $\left.E_{3}\right)$ can be obtained by the k.p theory by diagonalizing a strained $6 \times 6$ Hamiltonian of wurtzite symmetry. ${ }^{21}$ Including strain components along the three directions in the normal coordinate system (see Fig. 1 (a)) $\varepsilon_{x x}, \varepsilon_{y y}$, and $\varepsilon_{z z}$ and shear strain (only $\varepsilon_{x z}$ for simplicity) gives 


$$
\begin{aligned}
E_{1} & =\Delta_{c r}+\left(D_{1}+D_{3}\right) \varepsilon_{z z}+\left(D_{2}+D_{4}\right)\left(\varepsilon_{x x}+\varepsilon_{y y}\right)-D_{5}\left(\varepsilon_{x x}-\varepsilon_{y y}\right) \\
E_{2,3} & =\frac{1}{2} \Delta_{c r}+D_{1} \varepsilon_{z z}+D_{2}\left(\varepsilon_{x x}+\varepsilon_{y y}\right)+\frac{1}{2}\left[D_{3} \varepsilon_{z z}+D_{4}\left(\varepsilon_{x x}+\varepsilon_{y y}\right)+D_{5}\left(\varepsilon_{x x}-\varepsilon_{y y}\right)\right] \pm \frac{1}{2} \delta \\
\delta & =\sqrt{\left[\Delta_{c r}+D_{3} \varepsilon_{z z}+D_{4}\left(\varepsilon_{x x}+\varepsilon_{y y}\right)+D_{5}\left(\varepsilon_{x x}-\varepsilon_{y y}\right)\right]^{2}+8 D_{6}^{2} \varepsilon_{x z}^{2}} \\
\Delta E_{12} & =\frac{1}{2}\left[\Delta_{c r}+D_{3} \varepsilon_{z z}+D_{4}\left(\varepsilon_{x x}+\varepsilon_{y y}\right)\right]-\frac{3}{2} D_{5}\left(\varepsilon_{x x}-\varepsilon_{y y}\right)-\frac{1}{2} \delta
\end{aligned}
$$

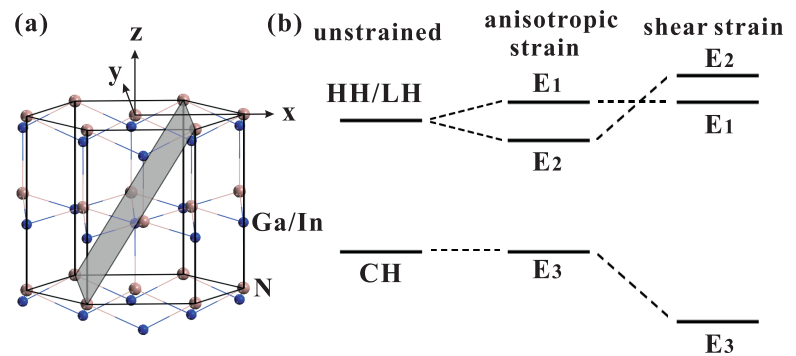

FIG. 1: (a) The normal coordinate system of the wurtzite GaN crystal. The $(11 \overline{2} 2)$ plane is shown in gray. (b) Schematic illustration of strain effects on the band structure in $(11 \overline{2} 2)$ semipolar InGaN. In an unstrained alloy, the heavy hole (HH) and light hole ( $\mathrm{LH})$ bands are doubly degenerate. Anisotropic strain in the $c$ plane causes the bands to split. Under shear strain, the $E_{2}$ band goes up and may switch order with the $E_{1}$ band.

where $\Delta_{c r}$ is the crystal-field splitting and the spin-orbit splitting has been omitted. The eigenstate pertaining to $E_{1}$ has $p_{y}$ character, and the other two have mixed $p_{x}$ and $p_{z}$ character. ${ }^{21} \Delta E_{12}$ gives the energy separation between the topmost two bands at the $\Gamma$ point. As can be seen from Eq. (1), anisotropic strain $\left(D_{5}\right)$ and shear strain $\left(D_{6}\right)$ have opposite effects because $D_{5}$ is negative. ${ }^{17}$ This is shown schematically in Fig. 1 (b). A switch in the band ordering may occur if the shear strain and/or the deformation potential $D_{6}$ are large enough.

If only shear strain is present the equations simplify to

$$
\begin{aligned}
& E_{1}=\Delta_{c r} \\
& E_{2,3}=\frac{\Delta_{c r}}{2} \pm \frac{\sqrt{\Delta_{c r}^{2}+8 D_{6}^{2} \varepsilon_{x z}^{2}}}{2} .
\end{aligned}
$$

Figure 2(a) shows the energies $E_{1}$ to $E_{3}$ as calculated in HSE06 for wurtzite GaN under shear strain. A similar plot is obtained for InN (not shown). As predicted by the $\mathbf{k} \cdot \mathbf{p}$ approach, the energy of one of the doubly degenerate valence bands remains constant under strain, while the other one increases. Concomitantly, the original crystal-field split-off band $(\mathrm{CH})$ goes down in energy. We then fit the energy separation between $E_{2}$ and $E_{3}$ $\left(\Delta E_{23}\right)$ shown in Fig. 2(b) to the expression above to obtain the deformation potential $D_{6}$. At the experimental lattice parameters, ${ }^{17}$ we obtain $D_{6}=-3.95 \mathrm{eV}$ for GaN and $D_{6}=-3.02 \mathrm{eV}$ for InN. These values are consistent
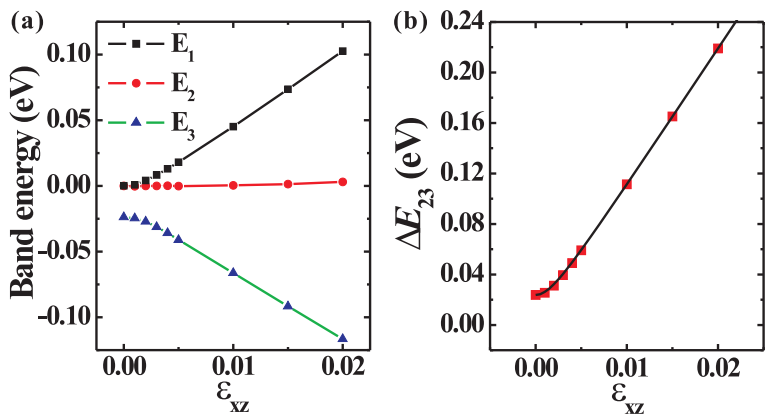

FIG. 2: (a) Energies of the topmost three valence bands of $\mathrm{GaN}$ under shear strain $\varepsilon_{x z}$. The valence-band maximum at zero shear strain is taken as energy zero. (b) Energy separation $\Delta E_{23}$ between valence bands $E_{2}$ and $E_{3}$.

with the general trend of our deformation potential set for the group-III nitrides ${ }^{17}$ in the sense that the magnitude of the deformation potentials is larger for GaN than for InN. Our $D_{6}$ value for $\mathrm{GaN}$ is smaller in magnitude than that reported by Vurgaftman and Meyer, ${ }^{22}$ which was obtained from other deformation potentials by means of the quasi-cubic approximation and not from direct measurements or calculations. For InN, our deformation potential is considerably smaller in magnitude than the value of $D_{6}=-8.8 \mathrm{eV}$ proposed by Ueda et al. ${ }^{11}$ which was deduced indirectly from the dependence of the polarization on indium composition.

Before we can investigate the effect of strain on the band structure the strain conditions in the alloy have to be determined. The lattice parameters follow from Vegard's law which simply averages over all local distortions: $a_{\mathrm{InGaN}}=a_{\mathrm{InN}} * x+a_{\mathrm{GaN}} *(1-x)$. The lattice mismatch to the GaN substrate induces composition-dependent compressive in-plane strain. If the InGaN layer thickness is below the critical value for misfit-dislocation formation, the lattice mismatch is accommodated by straining the layer elastically. The strain components in and out of the $c$ plane, which are needed for the $\mathbf{k} \cdot \mathbf{p}$ approach, can be determined from the lattice mismatch in the semipolar plane. ${ }^{23}$ Using the elastic constants of Wright ${ }^{24}$ we obtain the strain components for $(11 \overline{2} 2)$ semipolar quantum wells in terms of the normal $c$-plane coordinate system as shown in Fig. 3. We observe that these pseudomorphic 


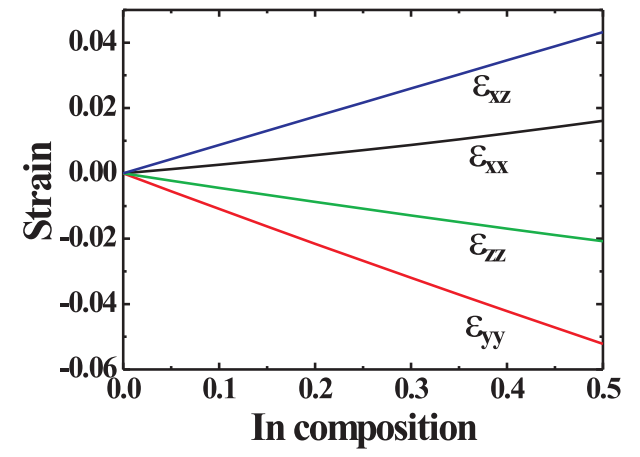

FIG. 3: Strain components in $(11 \overline{2} 2)$ semipolar InGaN grown on $\mathrm{GaN}$ as a function of In composition. $x, y$ and $z$ refer to the usual $c$-plane coordinate system.

strain conditions lead to anisotropic strain in the $c$-plane $\left(\varepsilon_{x x}-\varepsilon_{y y} \neq 0\right)$, and a large shear-strain component $\varepsilon_{x z}$.

To evaluate Eq. (1) for a given indium composition we linearly interpolate the deformation potentials of GaN and InN. In Fig. 4(a) we show the transition energies between the three valence bands and the conduction band, assuming that the (11̄22) InGaN layer is pseudomorphically grown on a GaN substrate. Under these conditions, the two highest valence bands do not cross over and the band ordering does not switch with increasing In concentration. The value of $\Delta E_{12}$ and the In concentration at which polarization switching occurs also depend on the degree of quantum confinement and on the spin-orbit splitting. Both are not included in our current model, but they do not change our qualitative results.

Due to the large lattice mismatch between $\mathrm{InN}$ and GaN (11\%), strains in alloys with high In content will be large and may be partially relaxed by forming structural defects (such as dislocations and stacking faults). According to Fig. 3, the magnitude of the strain component along the $y$ direction is larger than along the $x$ and $z$ directions. It is thus conceivable that the strain component in the $y$ direction would be partially relaxed in an InGaN layer grown on GaN. In Fig. 4(b) we plot $\Delta E_{12}$ for an $\operatorname{In}_{0.3} \mathrm{Ga}_{0.7} \mathrm{~N}$ alloy as a function of the strain components along the [1100] $(y)$ and [1123] (projection of $c$ axis on (11/22) plane) directions, expressed relative to their coherent value obtained from the lattice mismatch $\left[\epsilon_{0}=\left(a_{\text {substrate }}-a_{\text {alloy }}\right) / a_{\text {alloy }}\right]$. We find that $\Delta E_{12}$ becomes negative if the strain relaxation along [1100] is larger than that along [1123]. We conclude that partial strain relaxation may play a role in polarization switching of semipolar InGaN grown on GaN substrates.

Partial strain relaxation has indeed recently been ob- served in semipolar InGaN samples ${ }^{25}$ : The strain component along [1123] was found to experience a higher degree of relaxation through the formation of high-density dislocations facilitated by the existence of glide planes $(c$ plane) and the presence of shear strain. This relaxation pattern differs from the one that we predict to promote
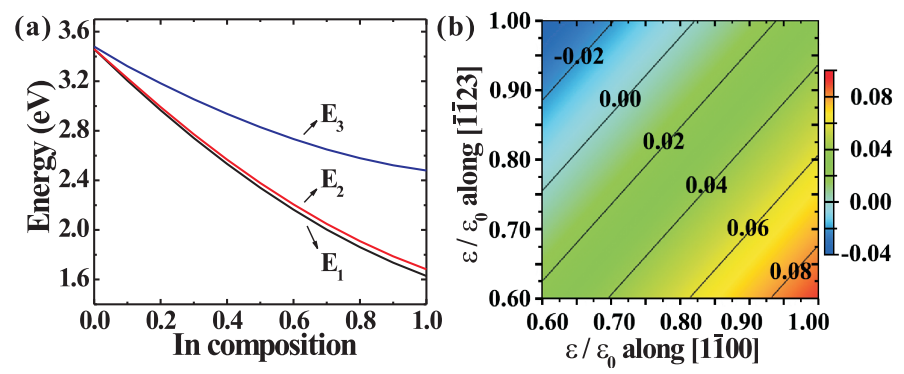

FIG. 4: Calculated transition energies between the conduction band and the topmost three valence bands of a (112̄2) semipolar InGaN alloy at the lattice parameters of $\mathrm{GaN}$ as a function of In composition. (b) Contour plot of the energy separation between the topmost two valence bands for $\operatorname{In}_{0.3} \mathrm{Ga}_{0.7} \mathrm{~N}$ as

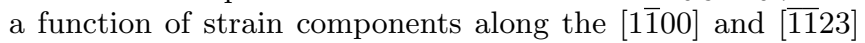
directions, expressed relative to their coherent value obtained from the lattice mismatch.

polarization switching [Fig. 4].

In conclusion, we have studied strain effects on the valence-band structure of $(11 \overline{2} 2)$ semipolar InGaN/GaN quantum wells. We have quantified the shear-strain deformation potential $D_{6}$ in wurtzite $\mathrm{GaN}$ and $\mathrm{InN}$ using first-principles HSE06 calculations. Using the $\mathbf{k} \cdot \mathbf{p}$ approach and our consistent set of deformation potentials we find that pseudomorphic strain conditions do not lead to polarization switching with increasing In concentration. A reduction of the strain component along the $y$ direction could lead to a band-ordering switch at In compositions around 0.3 , but such a relaxation pattern is not supported by experimental observations. ${ }^{25}$ We suggest that details of the band structure away from the zone center, along with band-filling effects, may play a role in the observed polarization switching.

This work was supported by the UCSB Solid State Lighting and Energy Center and by the Center for Energy Efficient Materials, an Energy Frontier Research Center funded by the U.S. DOE-BES under Award No. DESC0001009. It made use of the CNSI Computing Facility (NSF grant No. CHE-0321368) and TeraGrid computing resources (NSF grant No. DMR070072N). P. Rinke acknowledges the support of the Deutsche Forschungsgemeinschaft. We thank A. A. Yamaguchi, J. S. Speck, A. Romanov, A. Tyagi, and H. Masui for fruitful discussions.
1 S. Nakamura, Science 281, 956 (1998).

2 S. Pimputkar, J. S. Speck, S. P. Denbaars, and S. Nakamura, Nature Photonics 3, 180 (2009).
3 B. A. Haskell, F. Wu, S. Matsuda, M. D. Craven, P. T. Fini, S. P. DenBaars, J. S. Speck, and S. Nakamura, Appl. Phys. Lett. 83, 1554 (2003). 
${ }^{4}$ K. Nishizuka, M. Funato, Y. Kawakami, S. Fujita, Y. Narukawa, and T. Mukai, Appl. Phys. Lett. 85, 3122 (2004).

5 A. Tyagi, Y.-D. Lin, D. A. Cohen, M. Saito, K. Fujito, J. S. Speck, S. P. DenBaars, and S. Nakamura, Appl. Phys. Express 1, 091103 (2008).

${ }^{6}$ S. Ghosh, P. Waltereit, O. Brandt, H. T. Grahn, , and K. H. Ploog, Appl. Phys. Lett. 80, 413 (2002).

7 N. F. Gardner, J. C. Kim, J. J. Wierer, Y. C. Shen, and M. R. Krames, Appl. Phys. Lett. 86, 111101 (2005).

${ }^{8}$ H. Masui, H. Yamada, K. Iso, S. Nakamura, and S. P. Denbaars, J. Phys. D: Appl. Phys. 41, 225104 (2008).

${ }^{9}$ M. Ueda, K. Kojima, M. Funato, Y. Kawakami, Y. Narukawa, and T. Mukai, Appl. Phys. Lett. 89, 211907 (2006).

${ }^{10}$ H. Masui, T. J. Baker, M. Iza, H. Zhong, S. Nakamura, and S. P. DenBaars, J. Appl. Phys. 100, 113109 (2006).

11 M. Ueda, M. Funato, K. Kojima, Y. Kawakami, Y. Narukawa, and T. Mukai, Phys. Rev. B 78, 233303 (2008).

12 H. Masui, H. Asamizu, A. Tyagi, N. F. DeMille, S. Nakamura, and S. P. DenBaars, Appl. Phys. Express 2, 071002 (2009).

13 D. S. Sizov, R. Bhat, J. Napierala, C. Gallinat, K. Song, and C. en Zah, Appl. Phys. Express 2, 071001 (2009).
${ }^{14}$ K. Kojima, M. Funato, Y. Kawakami, S. Masui, S. Nagahama, and T. Mukai, Appl. Phys. Lett. 91, 251107 (2007).

15 A. A. Yamaguchi, Phys. Stat. Sol. (C) 5, 2329 (2008).

${ }^{16}$ P. Rinke, M. Winkelnkemper, A. Qteish, D. Bimberg, J. Neugebauer, and M. Scheffler, Phys. Rev. B 77, 075202 (2008).

17 Q. Yan, P. Rinke, M. Scheffler, and C. G. Van de Walle, Appl. Phys. Lett. 95, 121111 (2009).

18 G. Kresse and J. Furthmüller, Phys. Rev. B 54, 11169 (1996).

19 J. Heyd, G. E. Scuseria, and M. Ernzerhof, J. Chem. Phys. 118, 8207 (2003).

20 J. Heyd, G. E. Scuseria, and M. Ernzerhof, J. Chem. Phys. 124, 219906 (2006).

${ }^{21}$ G. L. Bir and G. E. Pikus, Symmetry and Strain-Induced Effects in Semiconductors (Wiley, New York, 1974).

${ }^{22}$ I. Vurgaftman and J. R. Meyer, J. Appl. Phys. 94, 3675 (2003).

23 A. E. Romanov, T. J. Baker, S. Nakamura, and J. S. Speck, J. Appl. Phys. 100, 023522 (2006).

24 A. F. Wright, J. Appl. Phys. 82, 2833 (1997).

25 A. Tyagi, F. Wu, E. C. Young, A. Chakraborty, H. Ohta, R. Bhat, K. Fujito, S. P. DenBaars, S. Nakamura, and J. S. Speck, Appl. Phys. Lett. 95, 251905 (2009). 